\title{
Performance of a PET Detector Module Utilizing an Array of Silicon Photodiodes to Identify the Crystal of Interaction
}

\author{
W.W. Moses, ${ }^{\dagger}$ S.E. Derenzo, ${ }^{\dagger}$ R. Nutt, ${ }^{\S}$ W.M. Digby, ${ }^{\S}$ C.W. Williams, ${ }^{\S}$ and M. Andreaco ${ }^{\S}$ \\ ${ }^{\dagger}$ Lawrence Berkeley Laboratory \\ University of California \\ Berkeley, California 94720 \\ $\S_{\text {CTI, Inc. }}$ \\ Knoxville, Tennessee 37933
}

November 1992

This work was supported in part by the Director, Office of Energy Research, Office of Health and Environmental Research, M lical Appiications and Biophysical Research Division, of the U.S. Department of Energy under Contract No. DE-AC03-76SF00098, in part by the National Institutes of Health, National Heart, Lung, and Blood Institute, National Cancer Insitute, and National Insitute of Neurological Disorders and Stroke under grants No. P01-HL25840, No. R01-CA48002, and No. R01-NS29655.

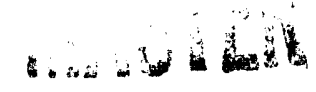




\section{PERFORMANCE OF A PET DETECTOR MODULE UTILIZING AN ARRAY OF SILICON PHOTODIODES TO IDENTIFY THE CRYSTAL OF INTERACTION*}

\section{Abstract}

We present initial performance results for a new multi-layer PET detector module consisting of an array of $3 \mathrm{~mm}$ square by $30 \mathrm{~mm}$ deep BGO crystals coupled on one end to a single photomultiplier tube and on the opposite end to an array of $3 \mathrm{~mm}$ square silicon photodiodes. The photomultiplier tube provides an accurate timing pulse and energy discrimination for the all the crystals in the module, while the silicon photodiodes identify the crystal of interaction. When a single $\mathrm{BGO}$ crystal at $+25^{\circ} \mathrm{C}$ is excited with $511 \mathrm{keV}$ photons, we measure a photodiode signal centered at 700 electrons $\left(\mathrm{e}^{-}\right)$with noise of $375 \mathrm{e}^{-}$fwhm. When a four crystal/ photodiode module is excited with a collimated line source of $511 \mathrm{keV}$ photons, the crystal of interaction is correctly identified $82 \%$ of the time. The misidentification rate can be greatly reduced and an $8 \times 8$ crystal / photodiode module constructed by using thicker depletion layer photodiodes or cooling to $0^{\circ} \mathrm{C}$.

\section{INTRODUCTION}

In the early 1980's it was demonstrated that under certain operating conditions, silicon photodiodes could detect the scintillation light from $511 \mathrm{keV}$ photon interactions in bismuth germanate (BGO) crystals with good signal to noise ratio and high efficiency $[1,2]$. Since then, a number of researchers have proposed PET detector modules that incorporate photodiodes $[3,4,5]$. However, achieving the requisite photodiode signal to noise ratio either severely degraded the performance of the module or increased the cost dramatically.

Silicon photodiode technology has improved dramatically in the last decade; in particular the dark current has been reduced by over two orders of magnitude. As a result, inexpensive silicon photodiodes can now be produced with the requisite signal to noise ratio. We report on the signal to noise ratio of one such photodiode operated under "normal" PET operating conditions and construct a simple, four detector element module that demonstrates the feasibility of a practical PET detector module that uses silicon photodiodes to determine the crystal of interaction.

\footnotetext{
* This work was supported in part by the U.S. Department of Energy under Contract No. DE-ACO3-76SFON098, and in part by Public Health Service Grant Nos. P01 25840, R01 CA48002, anu Rûi ivS29655.
}

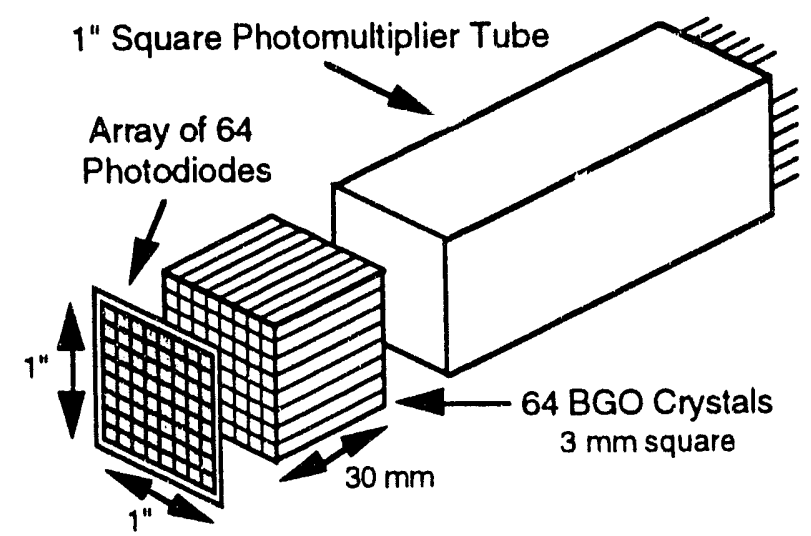

Figure 1: Exploded view of the proposed PET module. Each optically isolated $3 \times 3 \times 30 \mathrm{~mm}$ BGO crystal is attached to a $1^{\prime \prime}$ square photomultipher tube, which provides a timing pulse and energy discrimiration, and to an individual photodiode, which identifies the crystal of interaction

\section{BACKGROUND}

We propose the detector module shown in Figure 1 , which consists of a 8 by 8 array of optically isolated $3 \mathrm{~mm}$ square by $30 \mathrm{~mm}$ deep BGO crystals, each coupled on one $3 \times 3 \mathrm{~mm}$ face to a silicon photodiode and on the other $3 \times 3 \mathrm{~mm}$ face to a one inch square photomultiplier tube. The photomultiplier tube provides an accurate timing pulse and energy discrimination, while the photodiode array identifies the crystal of interaction. The photodiodes are read out with a VLSI array of 64 low noise charge sensitive amplifiers (not shown in Figure 1) that is mounted on the back (non-photosensitive) side of the photodiode array. This design takes advantage of the low cost and size of silicon photodiodes as well as the good timing and signal to noise ratio of photomultiplier tubes.

This module would be a factor of two to four smaller than existing block detector designs while utilizing the same number and size of photomultiplier tubes. This increases the maximum coincident event rate by two to four without increasing the cost due to photomultiplier tubes. The spatial resolution also improves, as individual coupling of scintillator crystals to photodetectors allows identification of events that Compton scatter in the detector module and removes the mispositioning error caused by statistical fluctuations in the light-sharing.

Similar designs have been proposed in the past, but involved prohibitive tradeoffs in price or performance. Previous results with PIN photodiodes only achieved the 


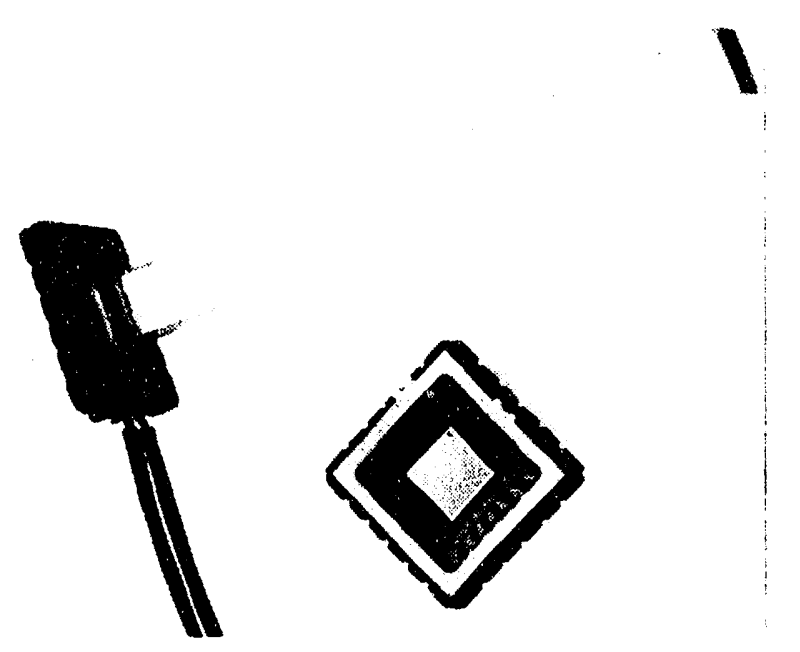

Figure 2: A portion of the single detector channel module. A $3 \times 3 \times 30 \mathrm{~mm}$ BGO crystal is attached to a $2.77 \mathrm{~mm}$ square active area photodiode. The $3 \times 30 \mathrm{~mm}$ sides of the crystal are wrapped with reflector material and the end opposite the photodiode is attached to a photomultiplier tube.

necessary signal to noise ratio when they were cooled to below $-40^{\circ} \mathrm{C}$, which decreases the shot noise associated with photodiode dark current and increases the BGO light output [6]. Cooling also increases the BGO decay time by an order of magnitude, and thus would increase the dead time of a PET tomograph by the same amount.

It has been suggested that the shot noise due to dark current at room temperature be reduced by using a higher band gap material such as mercuric iodide [5]. However, mercuric iodide's brittleness limits its reliability and none of the other high band gap materials (such as thallium bromide or cadmium telluride) has been manufactured with low enough dark current.

An alternative suggestion for improving the signal to noise is to use silicon avalanche photodiodes, which have an internal gain of approximately 100 [7]. These devices amplify the signal without significantly increasing the noise, but the cost of avalanche photodiodes is currently quite high. However, this is an attractive enough solution that one tomograph with these devices is currently under construction.

\section{SINGLE DETECTOR ELEMENT MODULE PERFORMANCE}

In order to predict the performance of the multielement detector module shown in Figure 1, it is necessary to know the signal and noise levels in a single element. To this end, we have constructed a simple module consisting of a single $3 \times 3 \times 30 \mathrm{~mm}$ BGO crystal coupled on one $3 \times 3 \mathrm{~mm}$ end to a photodiode and the other $3 \times$ $3 \mathrm{~mm}$ end to a $3 / 8$ inch square photomultiplier tube. A photograph of the BGO crystal coupled to the photodiode is shown in Figure 2, along with an unmounted photodiode. The remaining four $3 \times 30 \mathrm{~mm}$ sides of the BGO crystal were covered with white reflector material.

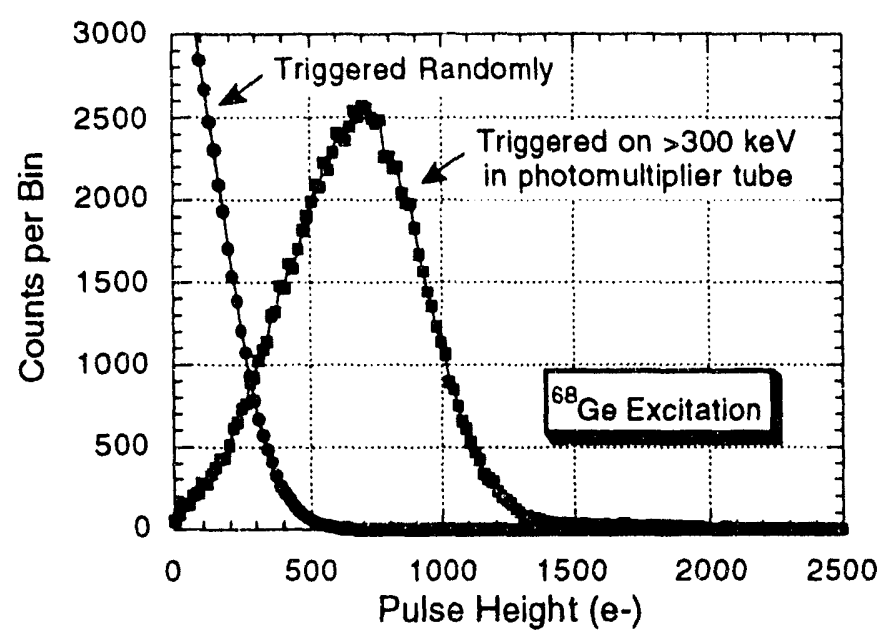

Figure 3: Pulse height spectrum measured in the photodiode when the detector is excited with $511 \mathrm{keV}$ photons and when triggered randomly. Operating temperature is $+25^{\circ} \mathrm{C}$ and amplifier shaping time is $2 \mu \mathrm{s}$.

The type of PIN photodiode used for all measurements presented herein is a Hamamatsu S-2506 mounted in a special package to allow close coupling to the scintillator crystal. The active rea of this device is $2.77 \mathrm{~mm}$ square, the depletion thickness is $100 \mu \mathrm{m}$, and the unit cost in large quantities is less than $\$ 1$. For this and all subsequent measurements, the photodiode was biased with $+30 \mathrm{~V}$ and the assembly operated at room temperature $\left(+25^{\circ} \mathrm{C}\right)$. Under these operating conditions, a typical capacitance was $9 \mathrm{pF}$ and dark current was $<100 \mathrm{pA}$.

The photodiode output signal is amplified with a low noise charge sensitive amplifier having a $2 \mu$ s shaping time. A calibrated test pulse injected into the front end of the amplifier was used to determine the dependence of the amplifier noise (in $\mathrm{e}^{-} \mathrm{fwhm}$ ) on detector capacitance and dark current, which were found to be:

$$
\text { Johnson Noise }\left(\mathrm{e}^{-} \text {fwhm }\right)=178+17.3 \mathrm{C} \quad(\mathrm{C} \text { in } \mathrm{pF})
$$
and

$$
\text { Shot Noise }\left(e^{-} f w h m\right)=2.35 * \sqrt{24 * I} \quad(I \text { in } p A),
$$

and the total noise is sum of these terms in quadrature, i.e.

$$
\text { Total Noise }=\sqrt{(\text { Johnson Noise })^{2 *}(\text { Shot Noise })^{2}} \text {. }
$$

This formula predicts a noise of $350 \mathrm{e}^{-}$fwhm for a S-2506 photodiode operated at room temperature, which agrees with the measured noise of $375 \mathrm{e}^{-}$fwhm.

The module was excited with $511 \mathrm{keV}$ photons from a ${ }^{68} \mathrm{Ge}$ source and the amplifier output digitized whenever the photomultiplier tube detected greater than $300 \mathrm{keV}$ energy deposit in the BCO crystal. A clear peak is visible in the resulting spectrum, which shown in Figure 3 . This peak is centered at a pulse height corresponding to 700 electrons $\left(e^{-}\right)$, and has a full-width at halfmaximum of $600 \mathrm{e}^{-}$. Fluctuations in the amount of energy deposited in the BGO crystal due to Compton interac- 
tions in the BGO crystal make this width larger than the $375 \mathrm{e}^{-}$due to electronic noise. When the radioactive source is removed and the photodiode amplifier output is digitized after the same number of random triggers, a noise peak that is well separated from the $511 \mathrm{keV}$ signal is observed, and is also shown in Figure 3.

\section{MONTE CARLO PREDICTION OF MODULE PERFORMANCE}

The performance of a single detector element can be extrapolated to predict the performance of a multielement module using a Monte Carlo simulation and determine whether the photodiode array has sufficient signal to noise ratio to identify the crystal of interaction. When a $511 \mathrm{keV}$ photon interacts in a module with $n$ detector elements, one clement should have a signal and the remaining $n-1$ elements should have only electronic noise. While the separation between the signal and noise in Figure 3 is large, there is significant overlap, so random fluctuations in the signal and noise levels can cause a "noise" detector element to have a larger pulse height than the "signal" detector element. In this case, the wrong element is identified as the crystal of interaction and mispositioning errors will degrade in the final image.

To predict the fraction of events in which this misidentification occurs, a Monte Carlo code was developed to simulate the interaction of the $511 \mathrm{keV}$ photon in the detector module, the resulting signal (and noise) in the photodiode amplifiers, and the assignment of the crystal of interaction. Three geometries were simulated an eight by eight array of $3 \times 3 \times 30 \mathrm{~mm}$ BGO crystals (64 crystals total), a six by six array of $4 \times 4 \times 30 \mathrm{~mm}$ BGO crystals ( 36 crystals total), and a two by two array of $3 \times 3$ x $30 \mathrm{~mm}$ BGO crystals (4 crystals total). The first two geometries simulated are full detector modules having a 1" $\times 1$ " $\times 30 \mathrm{~mm}$ volume of BGO, while the last geometry is a test module described in Section 5.

The simulation was performed by selecting a crystal near the center of the module to be the target crystal, and directing a normally incident $511 \mathrm{keV}$ photon toward a random point on the surface of this crystal. Annihilation photon absorption and detection in the BGO crystals was simulated using the energy dependent Compton and photoelectric effect cross sections, and the energy deposited in each detector crystal noted. If the total energy deposit in the module was less than $200 \mathrm{keV}$, the event was rejected. The conversion from energy deposit in each detector crystal to electron hole pairs at each amplifier input was done using the results from Section 3 (700 e- per $511 \mathrm{keV}$ energy deposit), and statistical fluctuations in this signal were also simulated. Electronic noise in each detector channel was simulated by adding Gaussian noise, and the element with the highest resulting output was selected as the crystal of interaction.

The fraction of events with the crystal of interaction correctly identified is shown in Figure 4 as a function of

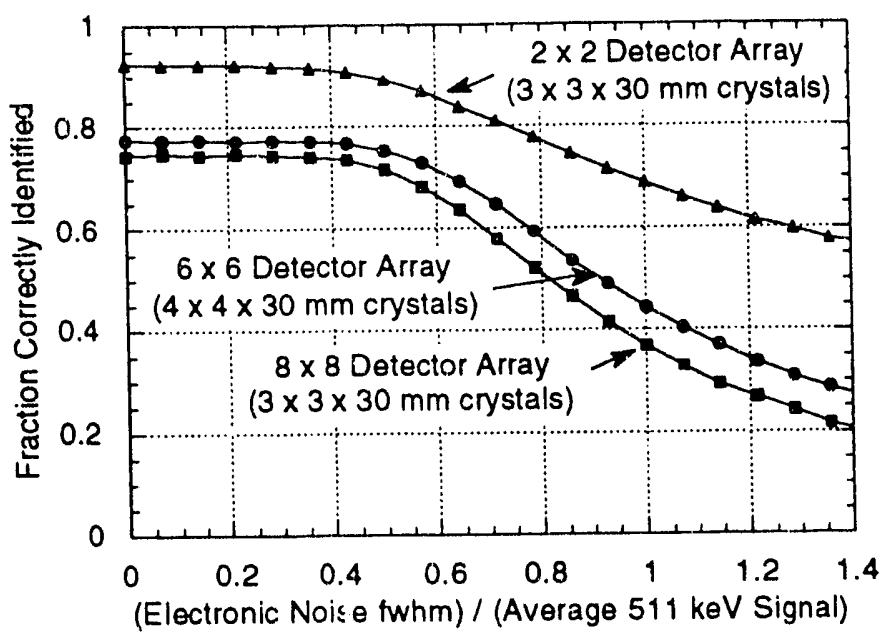

Figure 4: Fraction of correctly identified events as a function of the ratio of the photodiode amplifier electronic noise to the average signal seen per $511 \mathrm{keV}$ photon interaction. Three detector module geometries were simulated - a four crystal test module and full sized modules with 36 and 64 elements.

the value for electronic noise fwhm used in the simulation. In order to allow comparison to average signal levels other than $700 \mathrm{e}^{-}$per $511 \mathrm{keV}$ energy deposit, the noise level in Figure 4 is actually shown as a fraction of the average $511 \mathrm{keV}$ energy deposit signal. This is valid as long as the statistical fluctuations in the scintillation light output are smaller than the electronic noise, which is the case for any currently conceivable photodiode.

Figure 4 shows that even with zero electronic noise, some events are are misidentified due to Compton interactions within the detector block. This misidentification is decreased by using larger individual detector crystals (the probability of reabsorption within the same crystal increases) or by using a smaller overall detector module (the probability that the scattered photon leaves the block rather than interacting in another crystal within the block increases). As the electronic noise level increases, the misidentification rate stays constant until the noise fwhm becomes $40 \%$ of the average signal (roughly $300 \mathrm{e}^{-}$). The fraction of correctly identified events drops as the electronic noise is increased above $40 \%$ of the average signal, indicating that the electronic noise now causes additional events to be misidentified.

\section{FOUR DETECTOR ELEMENT MODULE PERFORMANCE}

To validate the Monte Carlo simulation in Section 4, we constructed and tested a prototype detector module consisting of a 2 by 2 array of $3 \mathrm{~mm}$ square by $30 \mathrm{~mm}$ deep BGO crystals coupled on one end to a single $3 / 8$ " square photomultiplier tube and on the opposite end to a 2 by 2 array of $2.77 \mathrm{~mm}$ square S-2506 silicon photodiodes. A photograph of the test module is shown in Figure 5 , along with an unmounted photodiode array. 

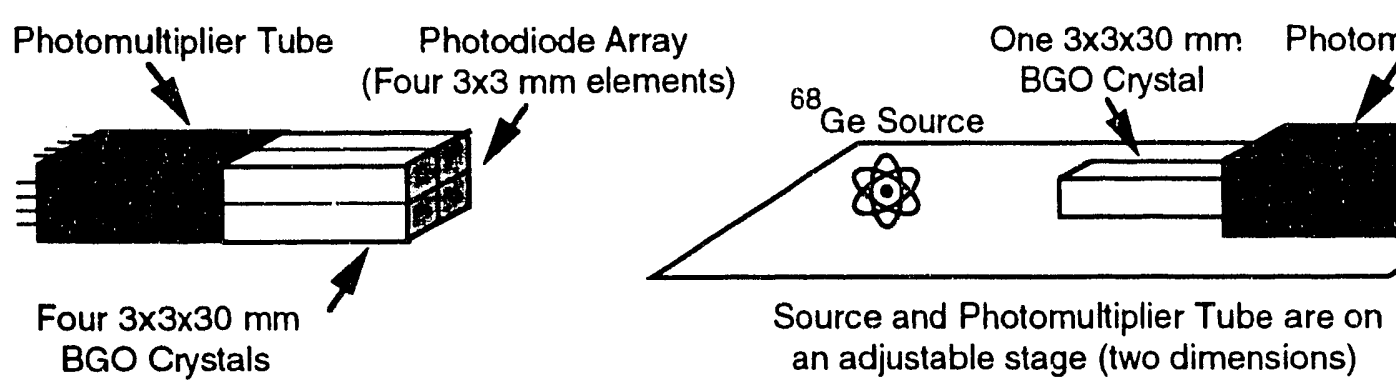

Figure 6: Experimental setup for measuring the fraction of events correctly identified. The single crystal and photomultiplier tube provide a $2 \mathrm{~mm}$ fwhm electrically collimated beam of $511 \mathrm{keV}$ photons that can be positioned with the adjustable stage.

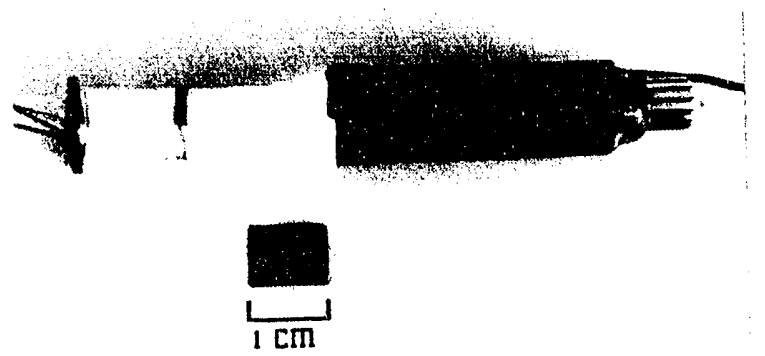

Figure 5: Test module with four $3 \times 3 \times 30 \mathrm{~mm} \mathrm{BGO}$ crystals attached to a $2 \times 2$ array of photodiodes. The crystals are wrapped with white reflector for optical isolation and the end opposite the photodiode attached to a photomultiplier tube.

This four crystal module was excited with a beam of $511 \mathrm{keV}$ photons that was electronically collimated ( $2 \mathrm{~mm}$ fwhm) using a single $3 \times 3 \times 30 \mathrm{~mm}$ BGO crystal coupled to a photomultiplier tube. A diagram of the detector module and the electronic collimation setup is shown in Figure 6. The collimated beam of $511 \mathrm{keV}$ photons was aligned with a crystal in the detector module. Whenever the photomultiplier tube detected an energy deposit greater than $250 \mathrm{keV}$ (in time coincidence with a similar energy deposit in the collimating photomultiplier tube), the four photodiode amplifiers were simultaneously digitized using four eight-bit flash ADCs. The channel with the greatest pulse height was then defined to be the crystal of interaction. There was no minimum photodiode signal required, so a crystal of interaction was assigned for each coincidence trigger.

Figure 7 shows the fraction of interactions assigned to the correct crystal. This figure shows an end-on view of the 2 by 2 array of square scintillator crystals. The upper left crystal is shaded to indicate thai it is aligned with the collimated beam of $511 \mathrm{keV}$ photons - the radiation symbol indicates the position of the center of the collimated beam. The numbers in each square represent the fraction of photomultiplier tube triggers in which that crystal was assigned the interaction - 10,000 total triggers were acquired. The crystal aligned with the $511 \mathrm{keV}$ photon beam was identified as the crystal of interaction $83 \%$ of the time, while the other three crystal were identified $4-6 \%$ of the time each.

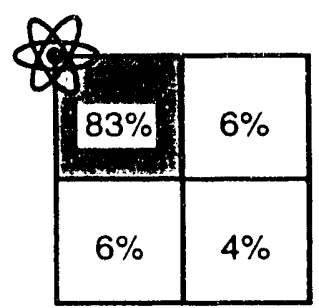

Figure 7: End view of test detector module showing the four crystals and the fraction of interactions assigned to each crystal. A $2 \mathrm{~mm}$ fwhm collimated beam of $511 \mathrm{keV}$ photons was centered on the upper left corner. Ideally, the shaded crystal should be $100 \%$ and the three unshaded crystals $0 \%$.

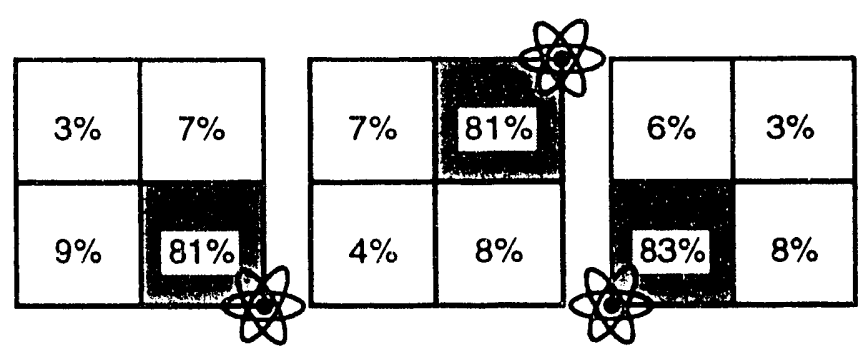

Figure 8: End view of the test detector module showing the fraction of interactions assigned to each crystal when the $2 \mathrm{~mm}$ fwhm collimated beam of $511 \mathrm{keV}$ photons was centered on the three other corners

The position of the collimated beam is moved and data collected at three other positions. The data, shown in Figure 8, show that the crystal aligned with the beam continues to be selected as the crystal of interaction $82 \%$ of the time. This agrees with the $82 \%$ predicted by the Monte Carlo results displayed in Figure 4.

\section{SUMMARY AND POSSIBLE IMPROVEMENTS}

The Monte Carlo simulation in Section 4 predicts that for the full 1" square module with an eight by eight array of $3 \times 3 \times 30 \mathrm{~mm}$ crystals to perform without crystal misidentification due to electronic noise, it must have an amplifier noise fwhm that is less $40 \%$ of the average $511 \mathrm{keV}$ energy deposit signal. The simulation is validated in Section 5. The single element tests in Section 2 show that an average $511 \mathrm{keV}$ energy deposit signal of 
$700 \mathrm{e}^{-}$is possible, which implies that an amplifier noise fwhm of less than $300 \mathrm{e}^{-}$is necessary.

As we currently achieve an amplifier noise fwhm of $375 \mathrm{e}^{-}$, some improvement is necessary before this proposed module can be realized. The most lit-cly place for improvement is the photodiode depletion thickness. The S-2506, with its $100 \mu \mathrm{m}$ depletion thickness, was selected for these tests partly because its $2.77 \mathrm{~mm}$ square geometry matched our detector geometry. We prefer a photodiode with $300 \mu \mathrm{m}$ depletion thickness, which would reduce the capacitance to $3 \mathrm{pF}$. Using the formulas for noise in Section 3, this yields an amplifier noise fwhm of $260 \mathrm{e}^{-}$, which is comfortably below the target of $300 \mathrm{e}^{-}$fwhm. Such photodiodes are readily available as custom orders.

Should the noise reduction from changing the photodiode depletion thickness prove insufficient, the average $511 \mathrm{keV}$ energy deposit signal could be increased by slight cooling. When $\mathrm{BGO}$ is cooled to $0^{\circ} \mathrm{C}$, its light output (and thus the signal) increases by a factor of 1.75 and its decay time increases by a factor of two (and so does the dead time) [8]. While the optimum operating temperature involves a tradeoff between crystal identification efficiency and dead time, it is very likely that an acceptable compromise can be reached.

\section{CONCLUSIONS}

A PET detector module that uses a room temperature array of silicon photodiodes to identify the crystal of interaction has been proposed. An average signal of $700 \mathrm{e}^{-}$per $511 \mathrm{keV}$ photon interaction and electronic noise of $375 \mathrm{e}^{-}$fwhm have been measured in a single detector element module. A Monte Carlo simulation predicts that crystal misidentification will not be affected by electronic noise as long as this noise fwhm is less than $40 \%$ of the average signal of $700 \mathrm{e}^{-}$per $511 \mathrm{keV}$ photon interaction (i.e. $300 \mathrm{e}^{-}$fwhm). A module consisting of a $2 \times 2$ array of detector elements was constructed and its ability to measure the crystal of interaction measured. While these measurements were slightly compromised by the finite size of the excitation beam of $511 \mathrm{keV}$ photons, they validated the Monte Carlo predictions. The devices used in these studies fell slightly short of the $40 \%$ signal to noise ratio needed to eliminate misidentification from electronic noise, but this target can be reached using $300 \mu \mathrm{m}$ depletion thickness photodiodes or by cooling the detector modules to no lower than $0^{\circ} \mathrm{C}$.

\section{ACKNOWLEDGEMENTS}

We would like to thank Mr. Tony Vuletich and Mr. Matt Ho of Lawrence Berkeley Lab for invaluable technical support. This work was supported in part by the Director, Office of Energy Research, Office of Health and Environmental Research, Medical Applications and Biophysical Research Division of the U.S. Department of Energy under contract No. DE-AC03-76SF00098, in part by the National Institutes of Health, National Heart,
Lung, and Blood Institute, National Cancer Institute, and National Institute of Neurological Disorders and Stroke under grants No. P01-HL25840, No. R01-CA48002, and No. R01-NS29655.

Reference to a company or product name does not imply approval or recommendation by the University of California or the U.S. Department of Energy to the exclusion of others that may be suitable.

\section{REFERENCES}

[1] Derenzo SE. Gamma-ray spectroscopy using small, cooled bismuth germanate scintillators and silicon photodiodes. Nucl. Instr. Meth. 219: pp. 117-122, 1984

[2] Groom DG. Silicon photodiode detection of bismuth germanate scintillation light. Nucl. Instr. Meth. 219: pp. $141-148,1984$.

[3] Barton JB, Hoffman EJ, Iwanczyk JS, et al. A highresolution detection system for positron tomography. IEEE Trans. Nucl. Sci. NS-30: pp. 671-675, 1983.

[4] Derenzo SE, Budinger TF and Vuletich $T$. High resolution positron emission tomography using narrow bismuth germanate crystals and individual photosensors. IEEE Trans. Nucl. Sci. NS-30: pp. $665-670,1983$.

[5] Dahlbom M, Mandelkern MA, Hoffman EJ, et al. Hybrid mercuric iodide $\left(\mathrm{HgI}_{2}\right)$-- gadolinium orthosilicate (GSO) detector for PET". IEEE Trans. Nucl. Sci. NS-32: pp. 533537,1985

[6] Derenzo SE. Initial characterization of a BGO-silicon photodiode detector for high resolution PET. IEEE Trans. Nucl. Sci. NS-31: pp. 620-626, 1984.

[7] Lecomte R, Cadorette J, Jouan A, et al. High resolution positron emission tomography with a prototype camera based on solid state scintillation detectors. IEEE Trans. Nucl. Sci. NS-37: pp. 805-811, 1990.

[8] Melcher CL, Schweitzer JS, Liberman, et al. Temperature dependence of fluorescence decay time and emission spectrum of bismuth germanate. IEEE Trans. Nucl. Sci NS-32: pp. $529-532,1985$. 

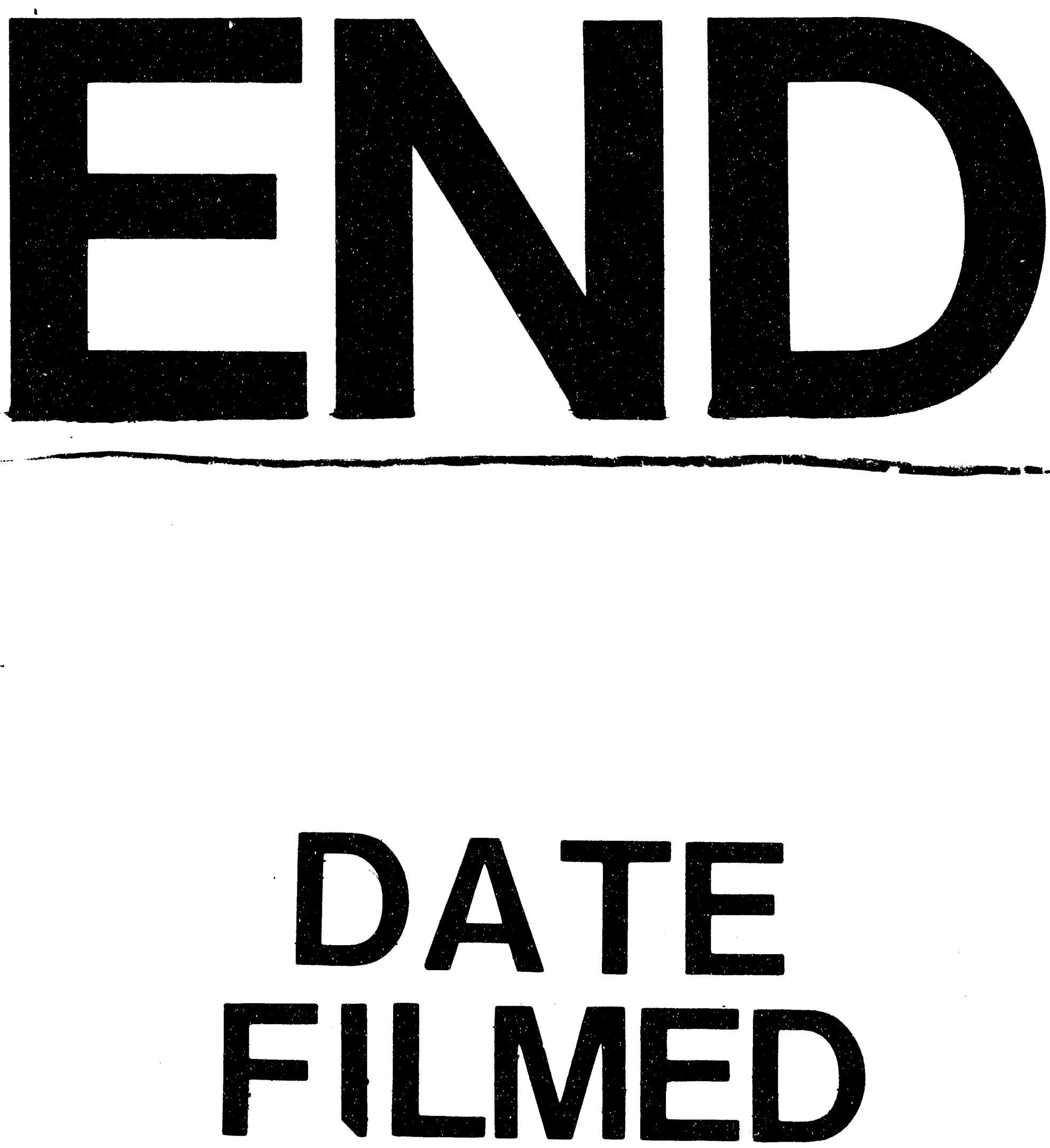

$$
5 / 14 / 93
$$


